TITLE:

\title{
Nuclear field shift effect in the isotope exchange reaction of cadmium using a crown ether
}

\section{$\operatorname{AUTHOR}(\mathrm{S})$ :}

Fujii, Toshiyuki; Moynier, Frédéric; Telouk, Philippe; Albarède, Francis

\section{CITATION:}

Fujii, Toshiyuki ...[et al]. Nuclear field shift effect in the isotope exchange reaction of cadmium using a crown ether. Chemical Geology 2009, 267(3-4): 157-163

\section{ISSUE DATE:}

2009-09

URL:

http://hdl.handle.net/2433/86912

\section{RIGHT:}

c 2009 Elsevier B.V. All rights reserved.; この論文は出版社版でありませ ん。引用の際には出版社版をご確認ご利用ください。; This is not the published version. Please cite only the published version. 
2 Nuclear Field Shift Effect in the Isotope Exchange Reaction of Cadmium Using a 3 Crown Ether

4

$5 \quad$ Toshiyuki Fujii ${ }^{1}$, Frédéric Moynier ${ }^{2}$, Philippe Telouk ${ }^{3}$, and Francis Albarède ${ }^{3}$

6

$7{ }^{1}$ Research Reactor Institute, Kyoto University, 2-1010 Asashiro Nishi, Kumatori, 8 Sennan Osaka 590-0494, Japan

$9{ }^{2}$ Department of Earth and Planetary Sciences, Washington University in St. Louis, 10 Campus Box 1169, 1 Brookings Drive, Saint Louis, MO 63130-4862, USA

$11{ }^{3}$ Laboratoire de Sciences de la Terre, UMR 5570 CNRS, Ecole Normale Supérieure de 12 Lyon, 46, Allee d'Italie, 69364 Lyon Cedex 7, France

16 *Author to whom correspondence should be addressed

17 tosiyuki@rri.kyoto-u.ac.jp

18 TEL: +81-724-51-2469, FAX: +81-724-51-2634

20 Keywords: Cadmium; Isotope fractionation; Mass-independent; Nuclear field shift; 21 Crown ether; Solvent extraction 


\section{Abstract}

24

25 Cadmium isotopes were fractionated by the liquid-liquid extraction technique with a

26 crown ether, dicyclohexano-18-crown-6. The isotopic ratios of ${ }^{\mathrm{m}} \mathrm{Cd} /{ }^{111} \mathrm{Cd}(\mathrm{m}$ : 110 ,

$27112,113,114$, and 116) were measured precisely by the multi-collector inductively

28 coupled plasma spectrometry (MC-ICP-MS). When the isotope enrichment factors

29 were calculated, the odd atomic mass isotopes $\left({ }^{111} \mathrm{Cd}\right.$ and $\left.{ }^{113} \mathrm{Cd}\right)$ showed excesses of

30 enrichment comparing to the even atomic mass isotopes $\left({ }^{110} \mathrm{Cd},{ }^{112} \mathrm{Cd},{ }^{114} \mathrm{Cd}\right.$ and $\left.{ }^{116} \mathrm{Cd}\right)$.

31 This odd-even staggering property originates from the nuclear field shift effect. The

32 contribution of the nuclear field shift effect to the observed isotope enrichment factor

33 was estimated to be 5 to $30 \%$. 


\section{Introduction}

37

The classic theory of chemical isotope fractionation only predicts mass-dependent isotope effect due to the isotopic difference in vibrational energies of isotopomers (Urey, 1947; Bigeleisen and Mayer, 1947). No exception to that rule was found until the mid-80s. Mass-independent isotope fractionations were first observed for O and S (see a review by Thiemens, 2006). The difference in the symmetry and the densities of states of the activated isotopomers is a possible origin for these mass-independent isotope

44 fractionations (Hathorn and Marcus, 1999). Alternative interpretations have been discussed in review articles (Weston, 1999; Thiemens, 2006). For heavy elements, an anomalous isotope enrichment of ${ }^{235} \mathrm{U}$ was found in a redox reaction using liquid

47 chromatography (Fujii et al., 1989). Isotope separation factors for even atomic mass 48 isotopes, ${ }^{234} \mathrm{U},{ }^{236} \mathrm{U}$, and ${ }^{238} \mathrm{U}$, showed a mass-dependent trend, while that of ${ }^{235} \mathrm{U}$

49 deviated from it. For middle-heavy elements, Nishizawa et al. (1995) found the anomalous isotope fractionation named the odd/even isotope effect, and a similarity

51 between the odd/even isotope effect and the odd-even staggering of $<r^{2}>$ were pointed 52 out (Nishizawa et al., 1995). To examine the mass-independent isotope effect, the 53 conventional mass-dependent theory was reconsidered by one of the original authors 54 (Bigeleisen, 1996). The original theory has been extended to include correction terms to account for nuclear properties, i.e., nuclear mass, nuclear size and shape, and nuclear spin. The nuclear field shift effect, which results from the isotopic change in the nuclear

57 size and shape, is recognized as the major origin of the mass-independent isotope effect 58 in uranium (Bigeleisen, 1996). 
60 Aufmuth et al., 1987) and molecular spectra (Tiemann et al., 1982). Basic knowledge of

61 isotope shifts is essential for understanding the isotope effects caused by the nuclear

62 properties. The field shift is an isotope shift which originates from the change of the

63 finite size and angular shape of the nuclear charge distribution when neutrons are added

64 to the nucleus. The isotope shifts result in a displacement in the minimum potential energy of oscillation curve of isotopomers (Bigeleisen, 1996).

The field shift is proportional to the isotopic difference in mean-squared

nuclear charge radius, $\delta<r^{2}>(\delta$ means isotopic difference) (King, 1984; Aufmuth et al., 1987). Mean-square radius, $\left\langle r^{2}>\right.$, of an odd atomic mass number isotope (with odd number neutrons) is usually smaller than the value expected from the adjacent isotopes with even atomic mass numbers (with even number neutrons). This unique property is known as the odd-even staggering which can be seen in every element (King, 1984; Aufmuth et al., 1987). The mass-independent isotope effect of uranium (Fujii et al., 1989) possesses the odd-even staggering property, and hence, the anomaly was attributable to the nuclear field shift effect (Bigeleisen, 1996; Nomura et al., 1996). element, zinc (Nishizawa et al., 1993, 1996, 1998a, 1998b; Fujii et al., 2001). Since employing macrocyclic polyethers in a chemical exchange method is effective to create 78 larger isotope fractionation (Tsuvadze et al., 1996), a crown ether was used as an extractant for a solvent extraction process (Nishizawa et al., 1993, 1996, 1998a) and a cryptand as a stationary phase for liquid chromatography (Nishizawa et al., 1998b; Fujii

81 et al., 2001). In every ligand exchange system, an odd atomic mass number isotope ${ }^{67} \mathrm{Zn}$

82 showed the anomalous enrichment property compared to that of the even atomic number 83 isotopes. From these results, we expect that the mass-independent isotope 
84 fractionation can also be found for a congener, cadmium. In this context, we

85 fractionated cadmium isotopes by using a liquid-liquid extraction with a crown ether,

86 dicyclohexano-18-crown-6 (DC18C6). The mass-independent property of $\mathrm{Cd}$ isotope

87 fractionation was investigated.

88 Cadmium isotopic anomalies have been found in few meteoric samples

89 (Rosman et al., 1978, 1980). Evaporation on the parent bodies of the meteorites is a

90 most probable origin for the Cd isotope fractionation (Rosman et al., 1978, 1980;

91 Wombacher et al., 2004, 2008). Wombacher et al. (2004) have studied the isotope

92 fractionation during evaporation experiments with a molten $\mathrm{Cd}$. This indicates that the

93 simple evaporation just causes the mass-dependent isotope fractionation of $\mathrm{Cd}$

94 (Wombacker et al., 2004, 2008). The nuclear field shift effect is considered as a possible

95 origin of isotopic anomalies found in several meteoritic samples (Fujii et al., 2006a,b).

96 Chemical reactions may have accompanied the formation of these samples, and hence, it

97 is interesting whether the mass-independent isotope fractionation occurs in our chemical

98 exchange system.

In studies of the $\mathrm{Zn}$ isotope effect (Nishizawa et al., 1993, 1996, 1998a, 1998b;

100 Fujii et al., 2001), isotopic compositions have been analyzed by ICP quadrupole mass

101 spectrometry (ICP-QMS) (Nishizawa et al., 1993), thermal ionization mass

102 spectrometry (TIMS) (Nishizawa et al., 1996, 1998a,), or multiple-collector ICP mass

103 spectrometry (MC-ICP-MS) (Nishizawa et al., 1998b; Fujii et al., 2001). In order to

104 obtain higher ionization yield, the silica gel method has been adopted for isotopic 105 analysis by TIMS (Nishizawa et al., 1996, 1998a,). In recent analytical studies on Zn

106 (Manhès and Göpel, 2003), Cd (Manhès and Göpel, 2003, 2007; Schmitt et al., 2006),

107 and $\mathrm{Pb}$ (Manhès and Göpel, 2003, 2007) isotopes, it has been pointed that a 
mass-independent bias may be created by using the silica gel method. Since the

110 ICP-MS, the anomaly should have been created in the chemical experiments, but the

111 pointed uncertainty of the silica gel method in TIMS should be taken care of. Hence, in

112 this study, we measured the Cd isotopic composition with a MC-ICP-MS.

\section{2. Experimental}

115 Dicyclohexano-18-crown-6 (over 97\% purity) and 1,2-dichloroethane (over 99.8\%

116 purity) were products of Fluka Chemie GmbH. Cadmium dichloride (hydrated,

117 99.999\% purity) was purchased from Sigma-Aldrich Co. Hydrobromic acid (twice

118 distilled) was purchased from Seastar Chemicals Inc. Hydrochloric acid and nitric

119 acid (Merck KGaA) of analytical grade were purified by distillation and supplied for 120 experiment.

121 Cadmium dichloride was dissolved in $\mathrm{HCl}$ to create solutions, $0.08 \mathrm{~mol} \mathrm{dm}^{-3}$ $122(\mathrm{M}) \mathrm{Cd}(\mathrm{II})$ in various $[\mathrm{HCl}](=1.6$ to $10 \mathrm{M})$. The organic phase was $0.1 \mathrm{M}$ DC18C6 in 123 1,2-dichloroethane. A $3 \mathrm{~cm}^{3}$ aqueous solution and a $3 \mathrm{~cm}^{3}$ organic solution were 124 mixed in a glass vial with a stirrer bar, and the glass vial was sealed with a stopcock.

125 The two phases were stirred by a magnetic stirrer for $30 \mathrm{~min}$. After the extraction 126 equilibrium was attained, the two phases were separated by centrifugation $(2000 \mathrm{rpm}, 1$

$127 \mathrm{~min})$. An aliquot of the upper aqueous solution was taken for analysis. These 128 procedures were carried out at $294.0 \pm 0.5 \mathrm{~K}$. The $\mathrm{Cd}$ concentration in the equilibrated aqueous phase was analyzed by ICP-QMS (Thermo Elemental X7). 
132 et al., 2006, 2007) on anion-exchange resin (AG1X8 200-400 mesh) in $\mathrm{HBr} / \mathrm{HNO}_{3}$.

133 An aliquot of the aqueous phase was once dried by heating at $333 \mathrm{~K}$ and then dissolved

134 into $1.5 \mathrm{M} \mathrm{HBr}$. On the anion-exchange resin in $1.5 \mathrm{M} \mathrm{HBr}, \mathrm{Cd}$ was strongly adsorbed

135 while organic materials went through. Finally, Cd was collected in $0.5 \mathrm{M} \mathrm{HNO}_{3}$.

136 A solution containing $200 \mathrm{ppb} \mathrm{Cd}$ in $0.05 \mathrm{M} \mathrm{HNO}_{3}$ was prepared for isotopic

137 analysis. Isotopic ratios of $\mathrm{Cd}$ in all samples were analyzed with the MC-ICP-MS Nu

138 plasma $500 \mathrm{HR}$ at ENS Lyon coupled with a desolvating nebulizer Nu DSN-100. 40

139 ratios in 2 blocks of 20 ratios each, in which the integration time of 1 scan was $10 \mathrm{sec}$,

140 were measured for each sample. The background was corrected for measuring the

141 ground zero at half-mass positions from the peak prior to data acquisition. The instrumental mass bias was corrected by bracketing each of the samples with standards.

143 The blank from the chemistry is $<1$ ng which is negligible with regards to the large

144 quantity of $\mathrm{Cd}$ extracted from the crown-ether experiments $(\sim 10 \mathrm{mg} \mathrm{Cd})$.

Possible interferences with Pd on the masses 108 and 110 and with In on the

146 mass 113 were verified to be under the detection of the ICP-MS. Because there was no

147 isotopic interference on ${ }^{111} \mathrm{Cd}$, the isotope ratios ${ }^{\mathrm{m}} \mathrm{Cd} /{ }^{111} \mathrm{Cd} \quad(m: 110,112,113,114$,

148 and 116) were measured. Since we were looking for odd-even effects we only selected

149 the more abundant even $\mathrm{Cd}$ isotopes (110, 112, 114 and 116) and did not measure the

150 least abundant ones, ${ }^{106} \mathrm{Cd}(1.25 \%)$ and ${ }^{108} \mathrm{Cd}(0.89 \%)$, which would not have give us

151 additional information. ${ }^{118} \mathrm{Sn}$ was measured to correct isobaric interferences of Sn with

$152 \mathrm{Cd}$ on the masses 112,114 , and 116. The interference of ${ }^{116} \mathrm{Sn}$ on ${ }^{116} \mathrm{Cd}$ as beam

153 intensities was less than $2 \times 10^{-4}$, and this was corrected by using the naturally occurring

154 isotopic abundances of Sn. The interferences of Sn on the masses 112 and 114 were

155 also corrected as the same manner, and were negligibly small (less than $4 \times 10^{-6}$ of ${ }^{112} \mathrm{Cd}$ 
156 or ${ }^{114} \mathrm{Cd}$ beam intensity).

\section{Results and discussion}

160 Possible species of $\mathrm{Cd}(\mathrm{II})$ in $\mathrm{HCl}$ media are $\mathrm{Cd}^{2+}, \mathrm{CdCl}^{+}, \mathrm{CdCl}_{2}, \mathrm{CdCl}_{3}{ }^{-}$, and $\mathrm{CdCl}_{4}{ }^{2-}$,

161 which are equilibrated as the following reactions,

162

$$
\mathrm{Cd}^{2+}+\mathrm{Cl}^{-} \leftrightarrow \mathrm{CdCl}^{+}
$$

$$
\mathrm{CdCl}^{+}+\mathrm{Cl}^{-} \leftrightarrow \mathrm{CdCl}_{2}
$$

$$
\mathrm{CdCl}_{2}+\mathrm{Cl}^{-} \leftrightarrow \mathrm{CdCl}_{3}^{-}
$$

164

$$
\mathrm{CdCl}_{3}{ }^{-}+\mathrm{Cl}^{-} \leftrightarrow \mathrm{CdCl}_{4}^{2-}
$$

166 Stability constants of equilibria 1 to 4 at zero ionic strength are evaluated from the

167 reported values, $\log K_{1}^{0}=2.0, \log K_{2}^{0}=0.70, \log K_{3}^{0}=-0.30$, and $\log K_{4}^{0}=-1.2$,

168 respectively (Pivovalov, 2005). From these $K^{0}$ values, activity of $\mathrm{Cl}^{-}$(Pivovalov, 169 2005), and mean activity of $\mathrm{CdCl}_{2}$ (Bromley, 1973), mole fractions of $\mathrm{Cd}$ species were estimated as functions of [HCl] (Figure 1). As shown in Figure 1, in our experimental

171 region of $[\mathrm{HCl}]=1.6$ to $10.0 \mathrm{M}$, the major species are $\mathrm{CdCl}_{2}, \mathrm{CdCl}_{3}{ }^{-}$, and $\mathrm{CdCl}_{4}{ }^{2-}$.

174 reaches a plateau. The extraction reaction of $\mathrm{Cd}$ in the crown ether system is generally

175 written as,

$$
\mathrm{Cd}^{2+}+2 \mathrm{Cl}^{-}+\mathrm{L} \leftrightarrow \mathrm{CdLCl}_{2}
$$

176 where L means ligand, DC18C6. The curve of $\log D$ vs. $\log a_{\mathrm{HCl}}$ can not be 
177 explained by this reaction. This acidity dependence would be attributable to a

178 co-extraction of $\mathrm{HCl}$. The extraction reaction may be rewritten as,

$$
n \mathrm{H}^{+}+\mathrm{CdCl}_{2}+n \mathrm{Cl}^{-}+\mathrm{L} \leftrightarrow \mathrm{CdLCl}_{2} \bullet n \mathrm{HCl}
$$

179 The stability constant of reaction $6, K_{\mathrm{L}}$, can be written,

$$
K_{\mathrm{L}}=\frac{\gamma_{\mathrm{CdLCl}_{2} \bullet n \mathrm{HCl}}\left[\mathrm{CdLCl}_{2} \bullet n \mathrm{HCl}\right]}{{a_{\mathrm{HCl}}{ }^{2 n}} \gamma_{\mathrm{CdCl}_{2}}\left[\mathrm{CdCl}_{2}\right] \gamma_{\mathrm{L}}[\mathrm{L}]}
$$

180

181 where we set the activity coefficients for the organic phase, $\gamma_{\mathrm{CdLCl}_{2} \bullet n H C l}$ and $\gamma_{\mathrm{L}}$, as

182 unities, because the condition of organic phase under the equilibrium would be quite

183 similar. Since $\left[\mathrm{CdLCl}_{2} \bullet n \mathrm{HCl}\right]$ can be replaced by $[\mathrm{Cd}(\mathrm{II})]_{\text {org }}$, the following equation is

184 obtained.

$$
\log [\mathrm{Cd}(\mathrm{II})]_{\mathrm{org}}-\log \gamma_{\mathrm{CdCl}_{2}}-\log \left[\mathrm{CdCl}_{2}\right]-\log [\mathrm{L}]=2 n \log a_{\mathrm{HCl}}+\log K_{\mathrm{L}}
$$

$185[\mathrm{Cd}(\mathrm{II})]_{\text {org }}$ and $[\mathrm{L}]$ are experimentally determined. $\gamma_{\mathrm{CdCl}_{2}}$ can be calculated by the 186 semi-empirical calculation (Bromley, 1973), and $\left[\mathrm{CdCl}_{2}\right]$ can be calculated from the

187 reported $K^{0}$ values (Pivovalov, 2005). As shown in Fig. 2, the left hand equation

188 shows a clear linearity to $\log a_{\mathrm{HCl}}$. From the slope and the intercept of this line

$189 K_{\mathrm{L}}=10.4$ and $n=0.7$ were determined, and the determination coefficient was $R^{2}=0.997$.

190 The obtained $n$ value suggests that $1 \mathrm{HCl}$ molecule may participate to the extraction

191 reaction 6. The co-extraction of $\mathrm{HCl}$ suggests that, in the organic phase, the extracted

$192 \mathrm{CdLCl}_{2} \bullet \mathrm{HCl}$ may form $\mathrm{H}^{+} \bullet \mathrm{CdLCl}_{3}{ }^{-}$. According to the reported extraction

193 stoichiometry of $\mathrm{H}^{+}$and anionic species (Beklemishev et al., 1997), the following

194 extraction may compete with the reaction 6 ,

$$
\mathrm{H}^{+}+\mathrm{CdCl}_{3}^{-}+\mathrm{L} \leftrightarrow \mathrm{HCdLCl}_{3}
$$

195 In a crown ether system, an extraction of anionic species from the concentrated $\mathrm{HCl}$ has 
196 been reported (Beklemishev et al., 1997). Thus, extraction of a neutral species

$197\left(\mathrm{CdLCl}_{2}\right)$, co-extraction of the neutral species and $\mathrm{HCl}$, and extraction of anionic species

198 with $\mathrm{H}^{+}$may simultaneously take place in the present system.

199

\subsection{Isotope fractionation during extraction}

202 is defined as:

203

$$
\alpha_{\mathrm{m}}=\frac{\left(\left[{ }^{\mathrm{m}} \mathrm{Cd}\right] /\left[{ }^{111} \mathrm{Cd}\right]\right)_{\mathrm{org}}}{\left(\left[{ }^{\mathrm{m}} \mathrm{Cd}\right] /\left[{ }^{111} \mathrm{Cd}\right]\right)_{\mathrm{aq}}}
$$

$204\left(\left[{ }^{\mathrm{m}} \mathrm{Cd}\right] /\left[{ }^{111} \mathrm{Cd}\right]\right)_{\text {org }}$ and $\left(\left[{ }^{\mathrm{m}} \mathrm{Cd}\right] /\left[{ }^{111} \mathrm{Cd}\right]\right)_{\text {aq }}$ are the isotope ratios of ${ }^{\mathrm{m}} \mathrm{Cd}$ relative to ${ }^{111} \mathrm{Cd}$

205 found in the organic and aqueous phases, respectively. Our recent study on mass-independent isotope effect fractionation of $\mathrm{Cr}$ created in the crown ether extraction system proved that the isotopic mass balance between two phases was well preserved (Fujii et al., 2008). Hence, we employ $\left(\left[{ }^{\mathrm{m}} \mathrm{Cd}\right] /\left[{ }^{111} \mathrm{Cd}\right]\right)_{\text {org }}$ calculated from $D$, $\left(\left[{ }^{\mathrm{m}} \mathrm{Cd}\right] /\left[{ }^{111} \mathrm{Cd}\right]\right)_{\mathrm{aq}}$ and $\left(\left[{ }^{\mathrm{m}} \mathrm{Cd}\right] /\left[{ }^{111} \mathrm{Cd}\right]\right)_{\text {init }}$ (the isotope ratios of ${ }^{\mathrm{m}} \mathrm{Cd}$ relative to ${ }^{111} \mathrm{Cd}$ in the

210 starting material). $\delta^{\mathrm{m}} \mathrm{Cd}$ is defined as

211

$$
\delta^{\mathrm{m}} \mathrm{Cd}=\left(\alpha_{\mathrm{m}}-1\right) \times 1,000
$$

213 As $\alpha_{\mathrm{m}}$ is almost equal to one, $\alpha_{\mathrm{m}}-1\left(=10^{-3} \delta^{\mathrm{m}} \mathrm{Cd}\right) \approx \ln \alpha_{\mathrm{m}}$.

214 The obtained $\delta^{\mathrm{m}} \mathrm{Cd}$ values are shown in Table 1. For each isotope, the

215 absolute value of $\delta^{\mathrm{m}} \mathrm{Cd}$ decreases and showed a minimum value at $5.2 \mathrm{M} \mathrm{HCl}$. Since the

216 dominant species in the aqueous phase are $\mathrm{CdCl}_{2}, \mathrm{CdCl}_{3}{ }^{-}$, and $\mathrm{CdCl}_{4}{ }^{2-}$ in the

217 experimental acidity region, possible isotope exchange reactions have taken place in 
reactions 2, 3, and 4 . The isotope exchange reactions have also taken place in the

219 extraction reactions 6 and 9. The acidity dependence of $\delta^{\mathrm{m}} \mathrm{Cd}$ we observed would be a

220 result of isotopic mass balance corresponding to these reactions.

The conventional mass-dependent theory of the Bigeleisen-Mayer equation

(1947) has been extended to include the nuclear field shift effect (Bigeleisen, 1996),

$$
\ln \alpha=\frac{h c}{k T} f_{s} \times a+\frac{1}{24}\left(\frac{h}{2 \pi k T}\right)^{2} \frac{\delta m}{m m} \times b
$$

where $f_{s}$ is the field shift, $a$ scaling factor for the nuclear field shift effect, $b$ the scaling factor for the vibrational mass effect. $m$ and $m$ ' are the masses of heavy isotope and light isotope, respectively, and $\delta m$ the difference of masses, $m-m$ '. Other symbols mean the usual physical constants. Since the field shift is proportional to the isotopic difference in mean-squared nuclear charge radius (King, 1984; Aufmuth et al., 1987), $\delta<r^{2}>$, the nuclear field shift effect is totally mass-independent.

The $\delta<r^{2}>_{\mathrm{m}}$ values of $\mathrm{Cd}$ (isotopic difference in mean-square radius, $\left\langle r^{2}>_{\mathrm{m}}-\right.$ odd-even staggering pattern. Similar trends can be seen in our data (Table 1), but magnitudes of the odd-even staggering seem to be smaller. This is because our $\varepsilon$ data are sum of mass-dependent and mass-independent isotope effects as shown in Eq. 12. We compare our $\varepsilon$ data with $\delta<r^{2}>$ data as follows.

237 while those for adjacent even ones can be expressed as $\delta^{2 \mathrm{n}} \mathrm{Cd}$ and $\delta^{2 \mathrm{n}+2} \mathrm{Cd}$. We 238 checked differences between $\left(\delta^{2 n+2} \mathrm{Cd}-\delta^{2 \mathrm{n}+1} \mathrm{Cd}\right)$ and $\left(\delta^{2 \mathrm{n}+1} \mathrm{Cd}-\delta^{2 \mathrm{n}} \mathrm{Cd}\right)$ by using data 239 given in Table 1. It should be noted that a correction with the reduced mass $(\mathrm{Sm} / \mathrm{mm}$ ') 
240 is required in order to compare these. The following ratio is evaluated.

241

$$
R_{\text {odd-even }}=\frac{\left(\delta^{2 n+1} C d-\delta^{2 n} C d\right) /\left(\frac{m_{2 n+1}-m_{2 n}}{m_{2 n+1} m_{2 n}}\right)}{\left(\delta^{2 n+2} C d-\delta^{2 n+1} C d\right) /\left(\frac{m_{2 n+2}-m_{2 n+1}}{m_{2 n+2} m_{2 n+1}}\right)}
$$

242 where $m_{2 \mathrm{n}+i}(i: 0,1$, or 2$)$ means mass of isotope with mass number $(2 \mathrm{n}+i)$. If $R_{\text {odd-even }}$

243 shows unity, this means that the observed isotope fractionation is totally

244 mass-dependent.

For isotope combinations, ${ }^{110} \mathrm{Cd}-{ }^{111} \mathrm{Cd}-{ }^{112} \mathrm{Cd}$ and ${ }^{112} \mathrm{Cd}-{ }^{113} \mathrm{Cd}-{ }^{114} \mathrm{Cd}, R_{\text {odd-even }} \mathrm{s}$

246 were calculated. The obtained $R_{\text {odd-even }}$ values are shown in Figure 4. In the same

247 manner with Eq. 13, $R_{\text {odd-even }}$ for $\delta<r^{2}>$ was also calculated, which is shown together.

248 The $R_{\text {odd-even }}$ values show clear deviation from unity. This proves that the isotope

249 fractionation of Cd contains the mass-independent isotope effect. The mass-independent

250 property may have been caused by the nuclear field shift effect.

In order to confirm that the nuclear field shift effect had taken place, we present isotopic anomalies in $\varepsilon$ unit. $\quad \varepsilon_{\mathrm{m}}$ is defined as parts per 10,000 deviation from

253 the mass dependent line drawn for a pair of even atomic mass isotopes, ${ }^{110} \mathrm{Cd}$ and ${ }^{114} \mathrm{Cd}$.

254 For comparison, the $\delta<r^{2}>$ values were also normalized by the same manner. Figure 5

255 shows a representative example for the $2.8 \mathrm{M}$ acidity case. As shown in Figure 5, the

256 normalized values for $\varepsilon$ show a quite similar trend to those for $\delta<r^{2}>$. Similar trends

257 were found for all acidity conditions. Hence, it can be concluded that the mass

258 independent isotope fractionation of $\mathrm{Cd}$ originated from the nuclear field shift effect. simplified as, 


$$
10^{-3} \delta^{\mathrm{m}} \mathrm{Cd}=\delta<r^{2}>_{\mathrm{m}} \times A+\frac{m-111}{m \times 111} \times B
$$

262 where $A$ and $B$ are also scaling factors. By applying Eq. 14 to the $\varepsilon_{\mathrm{m}}$ data, the scaling 263 factors $A$ and $B$ were determined for each acidity condition. Contributions of the 264 nuclear field shift effect, $\delta<r^{2}>_{\mathrm{m}} A / \varepsilon_{\mathrm{m}}$, and that of mass effect, $\{(m-111) / m \times 111\} B / \varepsilon_{\mathrm{m}}$, 265 were evaluated. These contributions on $\varepsilon_{\mathrm{m}}$ are shown in percent unit (Figure 6). The 266 contribution of the nuclear field shift effect (averaged value) was $5 \%\left(\delta^{110} \mathrm{Cd}\right)$, $26730 \%\left(\delta^{112} \mathrm{Cd}\right), 17 \%\left(\delta^{113} \mathrm{Cd}\right), 20 \%\left(\delta^{114} \mathrm{Cd}\right)$, or $17 \%\left(\delta^{116} \mathrm{Cd}\right)$, and no drastic change of this 268 ratio could be seen in the experimental acidity region. These contributions are 269 reproduced in Figure 7 as functions of $\delta \mathrm{m} / \mathrm{mm}$. The direction of the nuclear field shift 270 effect was same with that of the mass effect, and the magnitude of the mass effect was 271 larger than that of the nuclear field shift effect. This suppresses the mass-independent 272 property of the nuclear field shift effect. By increasing the nuclear field shift effect 273 and/or decreasing the mass effect, the mass-independent property will approach to the 274 profile of $\delta<r^{2}>$. Moreover, if the mass effect has a similar magnitude with an inverse 275 sign to the nuclear field shift effect, an intensive mass-independent property like Figure 2765 should appear. Under this extreme condition, the small change of the ratio of the 277 nuclear field shift effect vs. the mass effect results in the drastic change of the 278 mass-independent profile. There are two ways to change the ratio of the nuclear field shift effect $v s$. the

280 mass effect. The first way is to change the fractions of chemical species by controlling 281 acidity, ionic strength (concentrations of cation and anion and acidity), and 282 concentration of extractant. Since each isotopic exchange reaction (e.g., for reactions 
2831 to 6) may have a specific ratio of the nuclear field shift effect $v s$. the mass effect, this

284 ratio in the total isotope effect should be affected by the fractions of species, but, 285 changing acidity was less effective for the case of cadmium.

286 The second way is to change the molecular orbital itself by changing the

287 coordination and bonding circumstances, i.e., changing the types of extractant, counter 288 anion, aqueous and organic solvents. Investigating the second way should be 289 performed with a quantum chemical study on what kind of molecular orbital enlarges 290 the nuclear field shift effect. Recently, the magnitudes of nuclear field shift effects of 291 heavy elements ( $\mathrm{Tl}, \mathrm{Hg}, \mathrm{U}$, and so on) were estimated by employing quantum chemical 292 calculations (Schauble, 2007; Abe, 2008). The growth of their research field will be 293 helpful for further studies on nuclear field shift effect.

As a final note, we present a possibility of the nuclear field shift effect in nature.

295

\section{Conclusions}

Cadmium isotopes were fractionated by the liquid-liquid extraction technique with dicyclohexano-18-crown-6. The odd atomic mass isotopes showed excesses of 
307 enrichment comparing to the even atomic mass isotopes. This mass-independent

308 property is attributable to the nuclear field shift effect. The isotope enrichment factors

309 showed an acidity dependence, while the ratio of contributions of the mass effect and

310 the nuclear field shift effect were less sensitive to the acidity change.

311

312 Acknowledgment

313 TF thanks Roy Jacobus for his help in improving the English of this paper. The authors

314 thank two anonymous reviewers and the editors, Olivier Rouxel and Mathieu Roskosz,

315 for useful suggestions on the manuscript.

316 


\section{References}

Abe, M., Suzuki, T., Fujii, Y., Hada, M., 2008. An ab initio study based on a finite nucleus model for isotope fractionation in the U(III)-U(IV) exchange reaction system. J. Chem. Phys. 128, 144309.

Aufmuth, P., Heilig, K., Steudel, A., 1987. Changes in mean-square nuclear charge radii from optical isotope shifts. At. Data Nucl. Data Tables 37, 455-490.

Beklemishev, M. K., Dmitrienko, S. G., Isakova, N. V., 1997. Solvent extraction of metals with macrocyclic reagents and its analytical applications. in Macrocyclic Compounds in Analytical Chemistry, Zolotov, Yu. A., Ed., John Wiley \& Sons, New York.

Bigeleisen, J., Mayer, M. G., 1947. Calculation of equilibrium constants for isotopic exchange reactions. J. Chem. Phys. 15, 261-267.

Bigeleisen, J., 1996a. Nuclear size and shape effects in chemical reactions. Isotope chemistry of the heavy elements. J. Am. Chem. Soc. 118, 3676-3680.

Bromley, L. A., 1973, Thermodynamic properties of strong electrolytes in aqueous solutions. AIChE J. 19 (2), 313-320.

Fujii, T., Hirata, T., Shibahara, Y., Nishizawa, K., 2001. Mass-dependent and mass-independent isotope effects of zinc in chemical exchange reactions using liquid chromatography with a cryptand stationary phase. Phys. Chem. Chem. Phys. 3, 3125-3129.

Fujii, T., Moynier, F., Albarède, F., 2006a. Nuclear field shift effects as couse of isotopic anomalies in the early Solar System. Earth Planet. Sci. Lett. 247 (1-2), 1-9.

Fujii, T., Moynier, F., Telouk, P., Albarède, F., 2006b. Mass-independent isotope fractionation of molybdenum and ruthenium and the origin of isotopic anomalies in Murchison. Astrophys. J., 647, 1506-1516.

Fujii, T., Suzuki, D., Yamana, H., 2008. Nuclear field shift effect of chromium(III) in repeated extraction using a crown ether. Solvent Extr. Ion Exch. 26, 100-112.

Fujii, Y., Nomura, M., Okamoto, M., Onitsuka, H., Kawakami, F., Takeda, K., 1989a. An anomalous isotope effect of ${ }^{235} \mathrm{U}$ in $\mathrm{U}(\mathrm{IV})-\mathrm{U}(\mathrm{VI})$ chemical exchange. $\mathrm{Z}$. Naturforsch. A 44, 395-398.

Hathorn, B. C. Marcus, B. C., 1999. An intramolecular theory of the mass-independent isotope effect for ozone. I. J. Chem. Phys. 111, 4087-4100.

King, W. H., 1984. Isotope shifts in atomic spectra. Plenum Press, New York.

Manhès, G., Göpel, C., 2003. Heavy stable isotope measurements with thermal ionization mass spectrometry: Non mass-dependent fractionation effects between even and uneven isotopes. Geophys. Res. Abstr. 5, 10936-10936. 
Manhès, G., Göpel, C., 2007. Mass-independent fractionation during TIMS measurements: Evidence of nuclear shift effect? Geochim. Cosmochim. Acta. 71, A618-A618.

Moynier, F., Albarède, F., Herzog, G. F., 2006. Isotopic composition of zinc, copper, and iron in lunar samples. Geochim. Cosmochim. Acta 70, 6103-6117.

Moynier, F., Blichert-Toft, J., Telouk, P., Luck, J. M., Albarède, F., 2007. Comparative stable isotope geochemistry of $\mathrm{Ni}, \mathrm{Cu}, \mathrm{Zn}$, and $\mathrm{Fe}$ in chondrites and iron meteorites. Geochim. Cosmochim. Acta 71, 4365-4379.

Nishizawa, K., Nakamura, K., Yamamoto, T., Masuda, T., 1993. Zinc isotope effects in complex-formation with a crown-ether. Solvent Extr. Ion Exch. 11, 389-394.

Nishizawa, K., Satoyama, T., Miki, T., Yamamoto, T., Hosoe, M., 1995. Strontium isotope effect in liquid-liquid extraction of strontium chloride using a crown ether. J. Nucl. Sci. Technol. 32, 1230-1235.

Nishizawa, K., Satoyama, T., Miki, T., Yamamoto, T., Nomura, M., 1996. Separation of zinc isotopes by liquid-liquid extraction using a crown ether. Sep. Sci. Technol. 31, 2831-2841.

Nishizawa, K., Miki, T., Satoyama, T., Fujii, T., Yamamoto, T., Nomura, M., 1998a. Enrichment of zinc isotopes by a liquid membrane system using a crown ether. Sep. Sci. Technol. 33, 991-1002.

Nishizawa, K., Maeda, Y., Kawashiro, F., Fujii, T., Yamamoto, T., Hirata, T., 1998b. Contributions of nuclear size and shape, nuclear mass, and nuclear spin to enrichment factors of zinc isotopes in a chemical exchange reaction by a cryptand. Sep. Sci. Technol. 33, 2101-2112.

Nomura, M., Higuchi, N., Fujii, Y., 1996. Mass dependence of uranium isotope effects in the U(IV)-U(VI) exchange reaction. J. Am. Chem. Soc. 118, 9127-9130.

Pivovarov, S. 2005. Modeling of ionic equilibria of trace metals $\left(\mathrm{Cu}^{2+}, \mathrm{Zn}^{2+}, \mathrm{Cd}^{2+}\right)$ in concentrated aqueous electrolyte solutions at $25^{\circ} \mathrm{C}$. J. Colloid Interface Sci. 291, 421-432.

Rosman, K. J. R., De Laeter, J. R., 1978. A survey of cadmium isotopic abundances. J. Geophys. Res. 83, 1279-1287.

Rosman, K. J. R., Barnes, I. R., Moore, L. J., Gramlich., J. W., 1980. Isotope composition of $\mathrm{Cd}, \mathrm{Ca}$ and $\mathrm{Mg}$ in the Brownfield chondrite. Geochem. J., 14, 269-277.

Rosman, K. J. R., De Laeter, J. R., 1988. Cadmium mass fractionation in unequilibrated ordinary chondrites, Earth Planet. Sci. 89, 163-169.

Schauble, E. A., 2007. Role of nuclear volume in driving equilibrium stable isotope fractionation of mercury, thallium, and other very heavy elements, Geochim. Cosmochim. Acta 71, 2170-2189. 
391 Schmitt, A., Galer, S. J., Abouchami, W., 2006. Survey of natural cadmium isotope fractionation by double spike thermal ionization mass spectrometry. Eos Trans. AGU, 87, Fall Meet. Suppl., Abstract V12-A6.

394 Thiemens, M. H., 2006. History and application of mass-independent isotope effects. Annu. Rev. Earth Planet Sci. 34, 217-262.

396 Tiemann, E., Knöckel, H., Schlembach, J. 1982. Influence of the finite nuclear size on the electronic and rotational energy of diatomic molecules. Ber. Bunsenges. Phys. Chem. 86, 821-824.

399 Tsuvadze, A. Yu., Zhilov., V. I., Demin, S. V., 1996. Isotope separation with macrocyclic 400 polyethers. Russian J. Coord. Chem. 22, 229-237.

401 Urey, H. C., 1947. The thermodynamic properties of isotopic substances. J. Chem. Soc. $402 \quad 562-581$.

403 Weston, Jr., R. E., 1999, Anomalous or mass-independent isotope effects. Chem. Rev. 99, 2115-2136.

405 Wombacher, F., Rehkämper, M., Mezger, K., 2004. Determination of the 406 mass-dependence of cadmium isotope fractionation during evaporation. 68, $407 \quad 2349-2357$.

408 Wombacher, F., Rehkämper, M., Mezger, K., Bischoff, A., Münker, C. 2008. Cadmium stable isotope cosmochemistry. 72, 2349-2357.

410 
Table 1 Isotope fractionation of Cadmium.

\begin{tabular}{ccccccc}
\hline $\begin{array}{c}{[\mathrm{HCl}]} \\
/ \mathrm{M}\end{array}$ & $\begin{array}{c}\text { Number of } \\
\text { measurement }\end{array}$ & $\delta^{110} \mathrm{Cd}$ & $\delta^{112} \mathrm{Cd}$ & $\delta^{113} \mathrm{Cd}$ & $\delta^{114} \mathrm{Cd}$ & $\delta^{116} \mathrm{Cd}$ \\
\hline 2.8 & 5 & $1.19 \pm 0.14$ & $-1.48 \pm 0.06$ & $-2.64 \pm 0.09$ & $-4.07 \pm 0.06$ & $-6.51 \pm 0.11$ \\
4.0 & 2 & $1.21 \pm 0.14$ & $-1.40 \pm 0.06$ & $-2.62 \pm 0.09$ & $-3.95 \pm 0.07$ & $-6.35 \pm 0.17$ \\
5.2 & 5 & $0.12 \pm 0.04$ & $-0.14 \pm 0.02$ & $-0.23 \pm 0.05$ & $-0.37 \pm 0.06$ & $-0.59 \pm 0.17$ \\
6.4 & 6 & $0.31 \pm 0.09$ & $-0.38 \pm 0.01$ & $-0.71 \pm 0.02$ & $-1.07 \pm 0.03$ & $-1.71 \pm 0.06$ \\
7.6 & 3 & $0.63 \pm 0.14$ & $-0.76 \pm 0.06$ & $-1.40 \pm 0.09$ & $-2.11 \pm 0.07$ & $-3.40 \pm 0.17$ \\
8.8 & 6 & $0.28 \pm 0.02$ & $-0.33 \pm 0.02$ & $-0.62 \pm 0.04$ & $-0.93 \pm 0.03$ & $-1.50 \pm 0.04$ \\
\hline
\end{tabular}

423 Errors are $2 \sigma$ of experimental uncertainties (the error evaluation procedure can be seen 424 in our previous study (Fujii et al., 2006b).

425

426 
428 Figure 1 Formations of the Cd(II) species in HCl solutions. The reported stability constants for cadmium species (Pivovalov, 2005), the activity of $\mathrm{Cl}^{-}$(Pivovalov, 2005), and the mean activity of $\mathrm{CdCl}_{2}$ (Bromley, 1973) were used for calculation. The reported $a_{\mathrm{HCl}}$ (Bromley, 1973) was also used. Dissociation of $\mathrm{HCl}$ was treated as the complete dissociation. It should be noted that the thermodynamic data (Pivovalov, 2005; Bromley, 1973) used have been determined under 6 mol kg${ }^{-1}$ ionic strength.

434 Figure 2 Distribution ratios of $\mathbf{C d}(\mathrm{II}) . \quad D=[\mathrm{Cd}(\mathrm{II})]_{\mathrm{org}} /[\mathrm{Cd}(\mathrm{II})]_{\mathrm{aq}}$, in which $435[\mathrm{Cd}(\mathrm{II})]_{\text {org }}$ was determined as $[\mathrm{Cd}(\mathrm{II})]_{\text {init }}-[\mathrm{Cd}(\mathrm{II})]_{\mathrm{aq}}$. Since $\left[\mathrm{CdLCl}_{2} \bullet n \mathrm{HCl}\right]$ is much 436 smaller than $[\mathrm{HCl}]_{\text {init }}$, the consumption of $\mathrm{HCl}$ by the co-extraction was neglected.

437 The experimental acidities were $[\mathrm{HCl}]=1.6,2.8,4.0,5.2,6.4,7.6,8.8$, and $10.0 \mathrm{M}$, and 438 these were converted into $a_{\mathrm{HCl}}$ (Bromley, 1973). The unit of concentrations in right 439 ordinate is $\mathrm{mol} \mathrm{kg}^{-1}$.

440 Figure 3 Change in mean-square radius of $\mathbf{C d}, \delta<\boldsymbol{r}^{2}>_{\mathbf{m}} \cdot \delta<r^{2}>_{110}=-0.021 \pm 0.008$, $441 \delta<r^{2}>_{112}=0.119 \pm 0.024, \delta<r^{2}>_{113}=0.140 \pm 0.025, \delta<r^{2}>_{114}=0.244 \pm 0.032$, and $\delta<r^{2}>_{116}$ $442=0.342 \pm 0.037 . \delta<r^{2}>_{\mathrm{m}}$ values are calculated from reported data (Aufmuth et al, 1987).

443 Figure $4 \quad \boldsymbol{R}_{\text {odd-even }}$ values for isotope combinations ${ }^{110} \mathrm{Cd}-{ }^{111} \mathrm{Cd}-{ }^{112} \mathrm{Cd}$ and $444{ }^{112} \mathbf{C d}-{ }^{113} \mathbf{C d}-{ }^{114} \mathbf{C d}$. The thin dotted lines show $R_{\text {odd-even }}$ for $\delta<r^{2}>$. (See text for the 445 definition of $\left.R_{\text {odd-even }}\right)$.

446 Figure 5 Correlation between $\varepsilon$ and $\delta<r^{2}>$. Open marks show $\varepsilon_{\mathrm{m}}$ and solid marks $447 \delta<r^{2}>$. The data are normalized for an isotope pair ${ }^{110} \mathrm{Cd}$ and ${ }^{114} \mathrm{Cd}$. Errors of $\delta^{\mathrm{m}} \mathrm{Cd}$ 448 are drawn as those of $\varepsilon_{\mathrm{m}}$. For $\varepsilon_{111}$, the largest error $( \pm 1.4$, see Table 1$)$ found for $\delta^{\mathrm{m}} \mathrm{Cd}$ 449 was used.

450 Figure 6 Contributions of nuclear field shift effect and mass effect to observed 451 isotope enrichment factor. Open marks show the contribution of mass effect to $\delta^{\mathrm{m}} \mathrm{Cd}$ 452 and solid marks that to $\delta^{\mathrm{m}} \mathrm{Cd}$. These contributions were calculated by employing Eq. 14 .

453 Figure 7 Nuclear field shift effect, mass effect, and sum of these effects. Solid 454 circles show contributions of mass effect, while open circles those of nuclear field shift 455 effect. Open cross marks are sums of mass effects and nuclear field shift effects. These 456 contributions were calculated by employing Eq. $14 . \delta^{\mathrm{m}} \mathrm{Cd}$ is shown in arbitrary unit.

457 Figure 8 Isotopic variations of $\mathbf{C d}$ in type 3 ordinary condrites. a) Brownfield 458 (Rosman et al., 1980) b) Bishunpur (Rosman and De Laeter, 1988). Open marks show 
459 reported $\varepsilon_{\mathrm{m}} .{ }^{111} \mathrm{Cd}$ data of $\mathrm{b}$ ) was not reported. Solid marks show $\varepsilon_{\mathrm{m}}$ values estimated by 460 employing Eq. A11 in (Fujii et al., 2006a).

461 


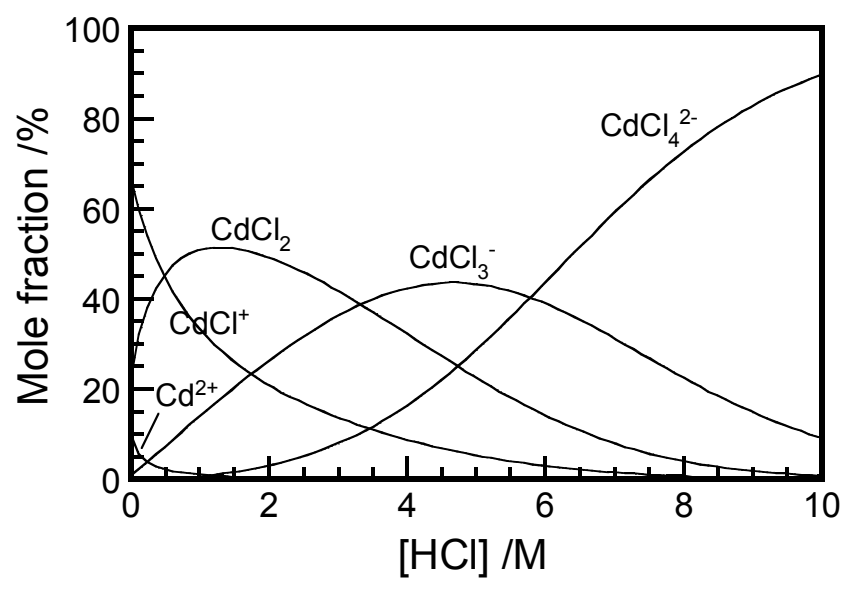




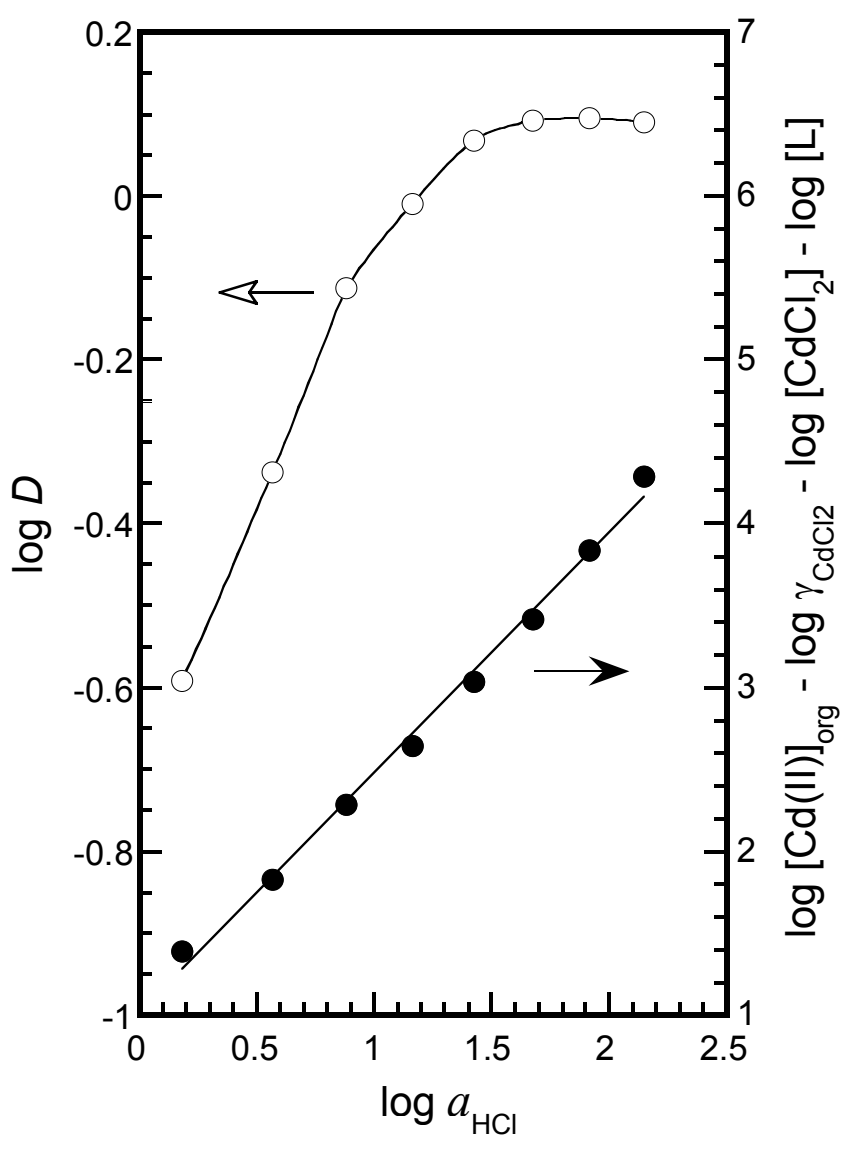




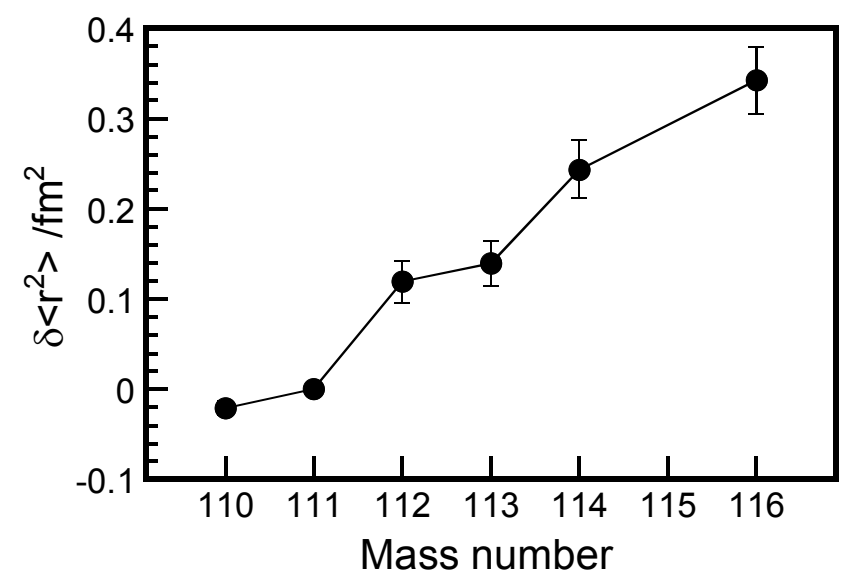




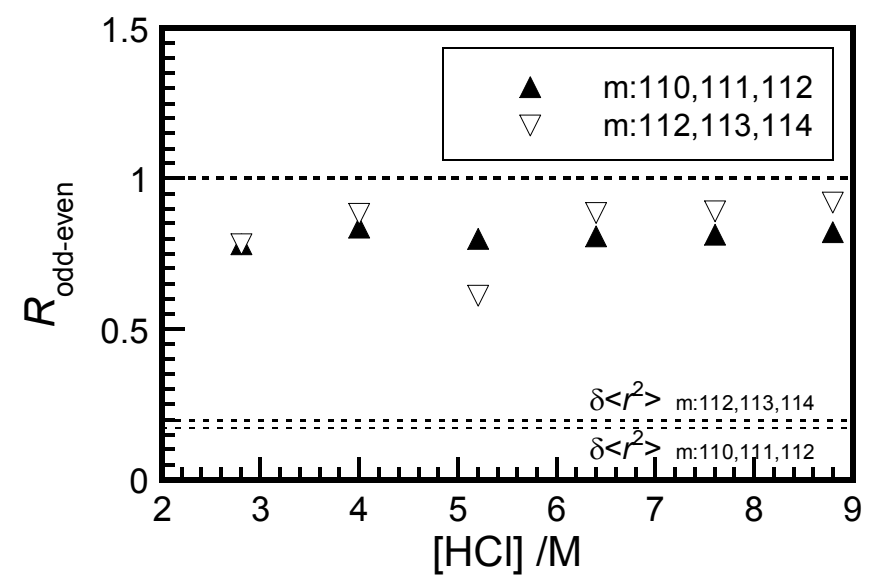




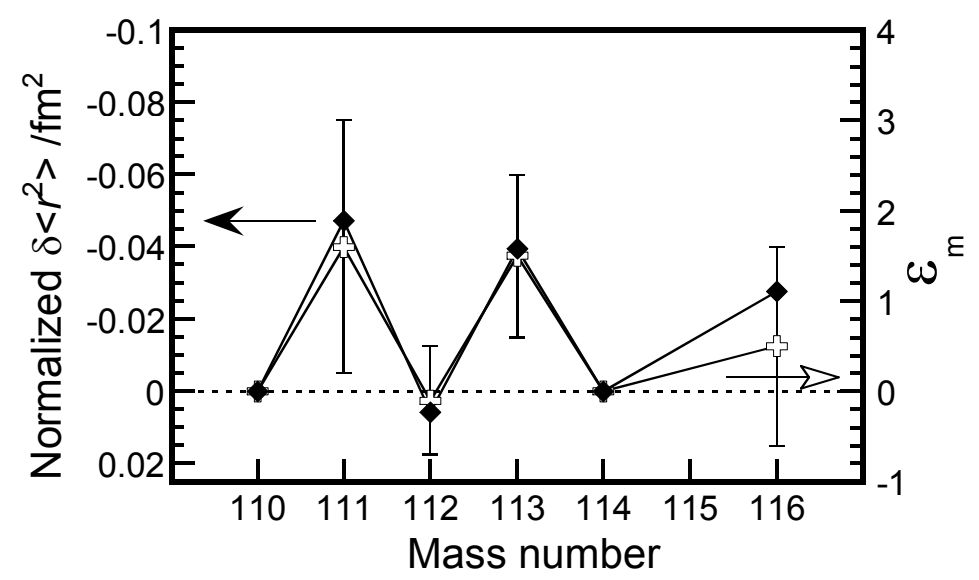



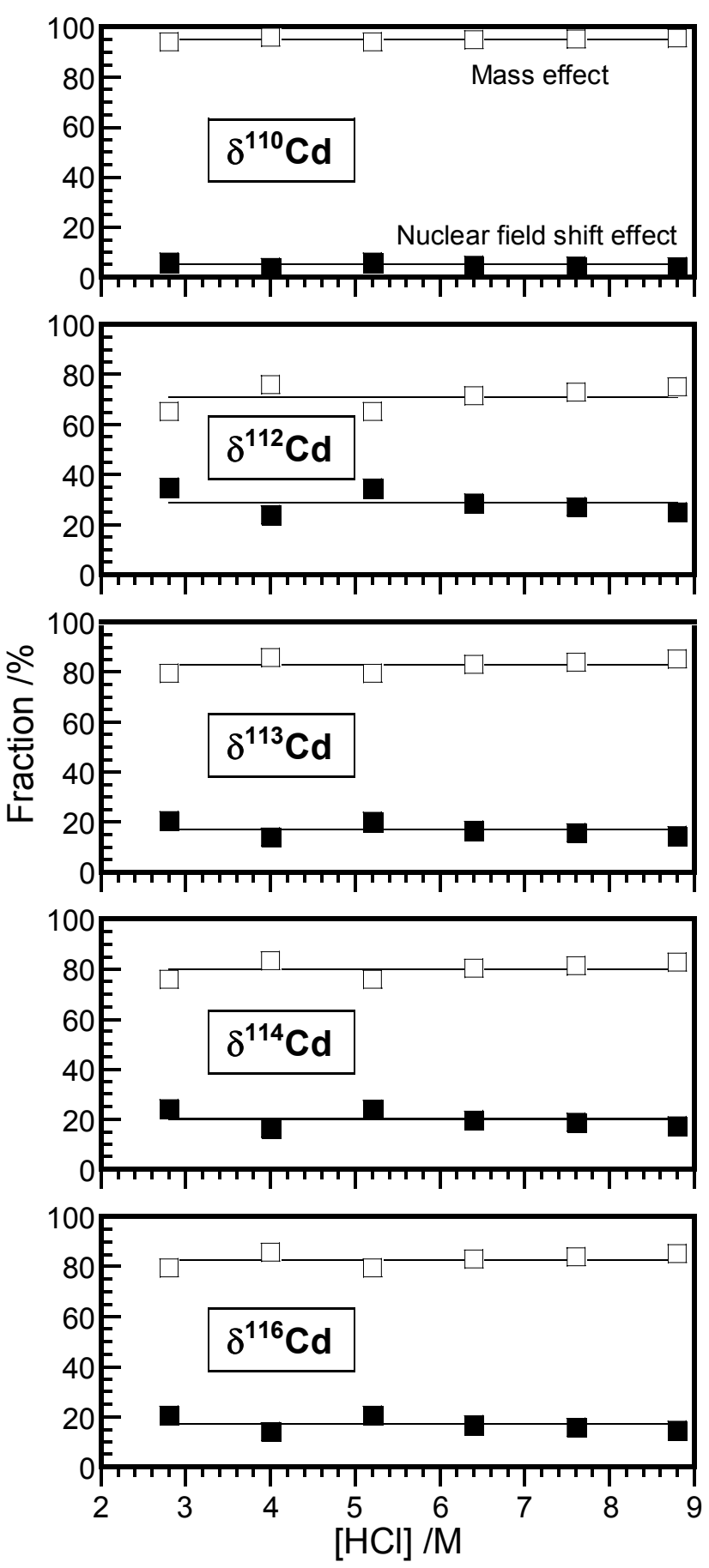


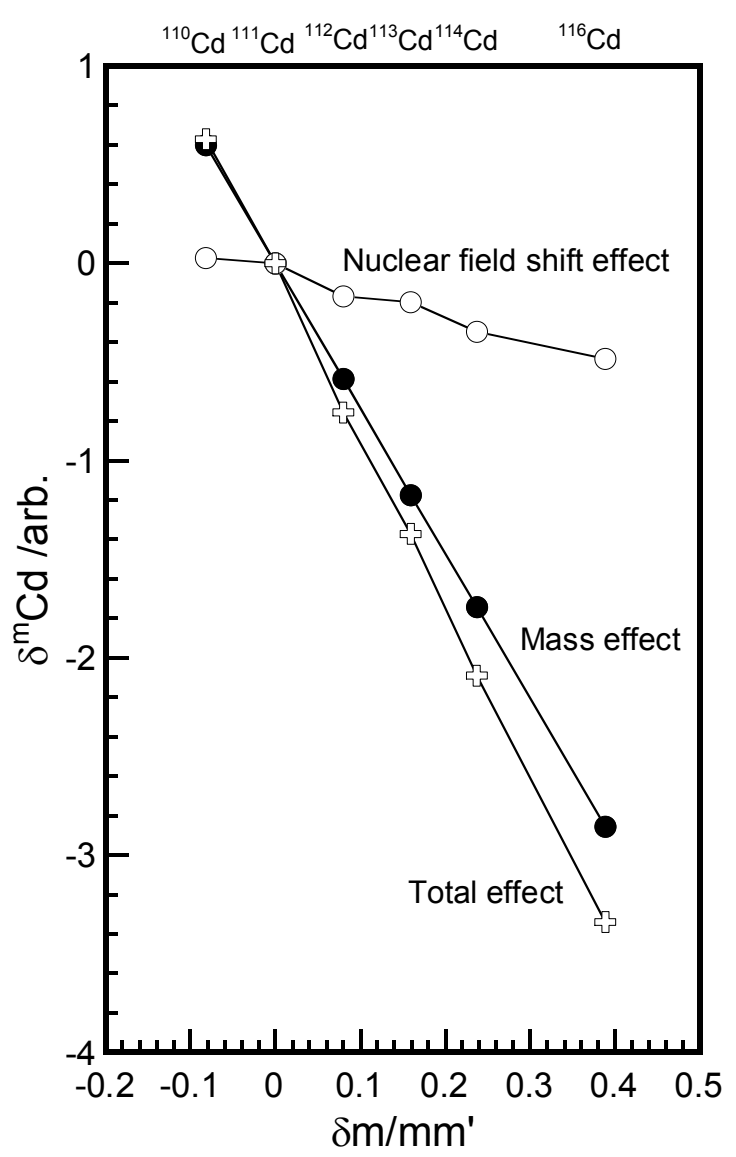



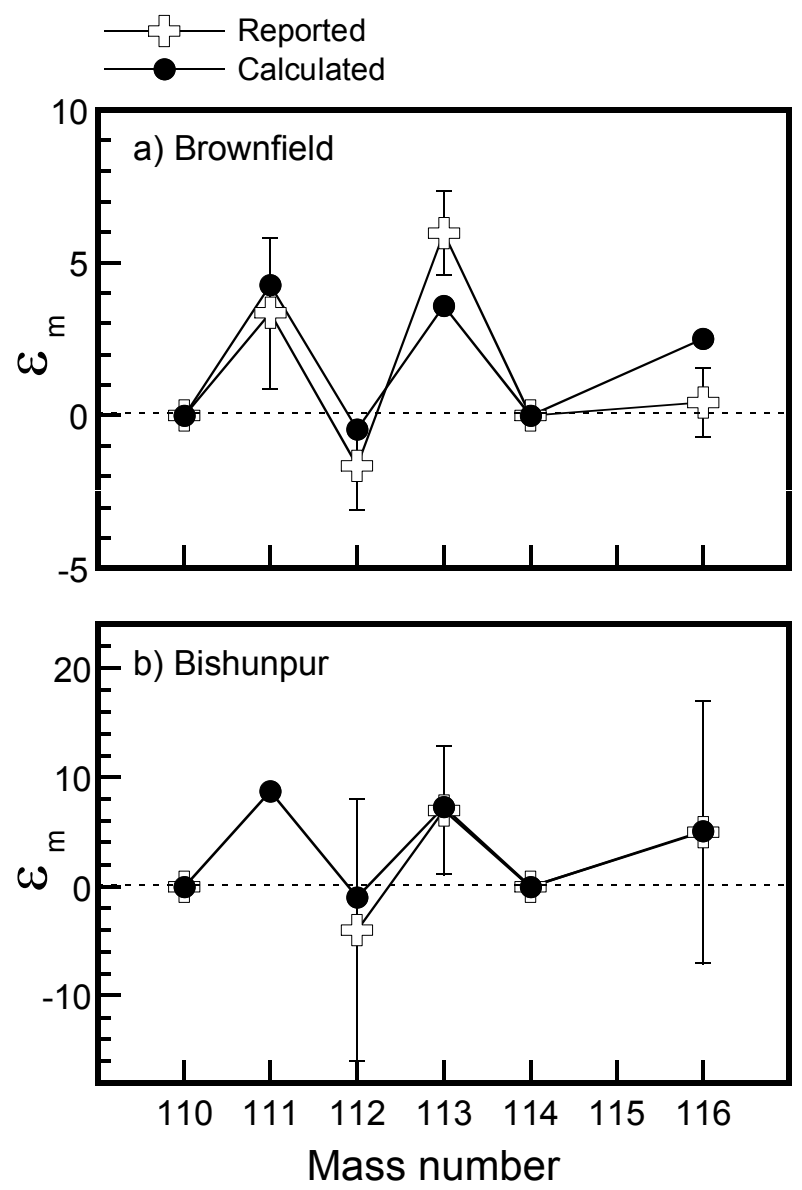